\title{
Effect of Monosaccharides and Disaccharides Type on Ionic Conductivity of Liquid Electrolyte Based Lithium Iodide
}

\author{
Nur Hani Ra'il', Nadhratun Naiim Mobarak ${ }^{1}$ \\ ${ }^{1}$ Department of Chemical Sciences, Faculty of Science and Technology, Universiti Kebangsaan Malaysia, 43600, Bangi, \\ Selangor Darul Ehsan, Malaysia
}

Article Info

Article History:

Received: March 18, 2021

Revised: April 24, 2021

Accepted: May 02, 2021

\section{Keywords: \\ liquid electrolyte monosaccharide disaccharide lithium iodide density functional theory (DFT)}

Corresponding Author:

Nadhratun Naiim Mobarak

Email:nadhratunnaiim@ukm.edu.my

\begin{abstract}
Liquid electrolyte was prepared by dissolving glucose, fructose, sucrose and lactose separately with different percentage of lithium iodide $(10-35 \%)$ in aqueous solution of $1 \%$ acetic acid. Liquid electrolyte is characterized using conductivity meter to determine ionic conductivity. Computer simulations of Density Functional Theory (DFT) was used to identify the dominant functional groups on monomers such as glucose, sucrose, fructose and lactose when interact with the lithium salt by using B3LYP/6-31G ++ $(d, p)$ basis set. The highest ionic conductivity for monosaccharide is glucose at $28.20 \mathrm{mS} / \mathrm{cm}$ while for disaccharide is lactose at $28.00 \mathrm{mS} / \mathrm{cm}$ with percentage of salt at $35 \mathrm{wt} . \%$. Ionic conductivity increases when concentration of salt increase because there is an interaction between salt with functional groups of compounds. Based on computer simulations of DFT, interaction between lithium with compounds can be occurred due to negative electrostatic potential on the molecule. Electronegativity value of oxygen atom in glucose $(-0.562 \mathrm{e})$ and lactose ($0.567 \mathrm{e})$ higher than fructose $(-0.559 \mathrm{e})$ and sucrose $(-0.515 \mathrm{e})$. Functional groups that are dominant to interact when interact with lithium salt are O-15 for glucose and $\mathrm{O}-17$ for lactose due to the shorter bond length, the stronger energy attraction between functional groups with lithium.
\end{abstract}

\section{INTRODUCTION}

Natural polymers such as starch, cellulose, chitosan or carrageenan are built from monomers glucose, glucosamine or fructose. According to Khanmirzaei and Ramesh (2013), natural polymers have economic and environmental benefits due to cheap in cost and biodegradability. Natural polymers have greatly involved in many applications such as solar cells, lithium batteries and fuel cells. Based on previous studies that used starch (Khanmirzaei \& Ramesh, 2013), carrageenan (Mobarak et al., 2012) and chitosan (Aziz \& Abidin, 2013) showed that natural polymers can be used as main polymer in polymer electrolyte system.

According to the studies by Liu et al. (2016), it is proved that natural compound like sucroseboron (B-sucrose) can give excellent electrochemical performance. In addition, previous work by Khanmirzaei and Ramesh (2013) also showed natural polymers likes starch can provide the highest ionic conductivity of $4.68 \times 10^{-5} \mathrm{~S} \mathrm{~cm}^{-1}$ with a percentage of salt is $35 \mathrm{wt} . \%$. The analysis of ionic conductivity for electrolyte system can be measured because of interaction between ions with host polymer. It can be 
confirmed by using Fourier Transform Infrared (FTIR) spectroscopy analysis that shown the shifted of functional group which is hydroxyl group on the FTIR spectra.

Polysaccharides are high molecular weight carbohydrate molecules composed of several monosaccharide units. One unit of polysaccharides like cellulose and starch, it has three hydroxyl groups on the monomer. In addition, each monomer in polysaccharides linked by ether groups (Wade 2014). A computational approach using the Density Functional Theory (DFT) calculation was used to identify the dominant functional groups on monosaccharide units such as glucose, sucrose, fructose and lactose when interact with the lithium salt. Therefore, to support our experimental of ionic conductivity study, we present simulation on the interaction of monomers units with lithium using DFT calculation.

Polysaccharide monomers vary structurally and stereochemically. They have different ring structures. For example, polysaccharides such as carrageenan is composed of five membered ring and six membered ring while polysaccharides such as starch and cellulose composed of six membered ring monomers. In previous studies, Selvin et al. (2018) was used K-carrageenan as the host polymer while $\mathrm{NH}_{4} \mathrm{SCN}$ has been chosen as the ionic dopant. The highest ionic conductivity that can be obtained is $6.83 \times 10^{-4} \mathrm{~S} \mathrm{~cm}^{-1}$. The studies by Zulkefli et al. (2015) on polymer electrolyte based starch as host polymer and $\mathrm{NH}_{4} \mathrm{SCN}$ salt as the ionic dopant shown that the highest ionic conductivity is $5.54 \times 10^{-3}$ $\mathrm{S} \mathrm{cm}^{-1}$. As a consequence, the aim of this research is to discover the influence of ring type that built a polysaccharide can affect the ionic conductivity of polymer electrolyte system.

\section{METHOD}

\subsection{Materials}

Glucose $\left(\mathrm{C}_{6} \mathrm{H}_{12} \mathrm{O}_{6}\right)$ (Sigma Aldrich), fructose $\left(\mathrm{C}_{6} \mathrm{H}_{12} \mathrm{O}_{6}\right)$ (Sigma Aldrich), sucrose $\left(\mathrm{C}_{12} \mathrm{H}_{22} \mathrm{O}_{11}\right)$ (Sigma Aldrich), lactose $\left(\mathrm{C}_{12} \mathrm{H}_{22} \mathrm{O}_{11}\right)$ (Sigma Aldrich), acetic acid (Sigma Aldrich) and lithium iodide salt (Sigma Aldrich, crystalline powder, 99.9\% trace metal basis) have been used for sample preparation.

\subsection{Preparation of liquid electrolyte}

Glucose, fructose, sucrose and lactose were dissolved respectively into different amounts of weight percent of lithium iodide in acetic acid and stirred continuously until the complete dissolution of the salt.

\subsection{Characterization method}

\subsubsection{Ionic conductivity}

Ionic conductivity study was carried out using ionic conductivity meter, Mettler Toledo at constant temperature, $25^{\circ} \mathrm{C}$.

\subsubsection{Computational methods}

The molecular structures of glucose, fructose, sucrose and lactose were optimized by the density functional theory (B3LYP) method with the 6-31G ++ (d, p) basis set, using Gaussian 09 package program and Gaussview molecular visualization program. The other calculations were performed by using Becke's three parameter functional (B3), combined with gradient corrected correlational functional of Lee, Yang and Parr (LYP), supplemented with standard 6-31G ++ (d, p) basis set.

\section{RESULTS AND DISCUSSION}

\subsection{Ionic conductivity}

Ionic conductivity of electrolytes depends upon charge carrier concentration, charge on carrier and size of carrier. Hence, when charge concentration is change, the conductivity is also expected to change. Figure 1 illustrates the variation of ionic conductivity with different weight $(1.0-3.5 \mathrm{~g})$ of glucose, fructose, sucrose and lactose with the same concentration of salt (5 wt.\%). Figure 1 showed 
that conductivity increased with addition of weight for each compound respectively. This is because increased in weight compounds, had increased the active site in the electrolyte system and it gives more room for the cations or protons to interact (Imperiyka et al., 2014). Thus, it is increased in ionic conductivity.
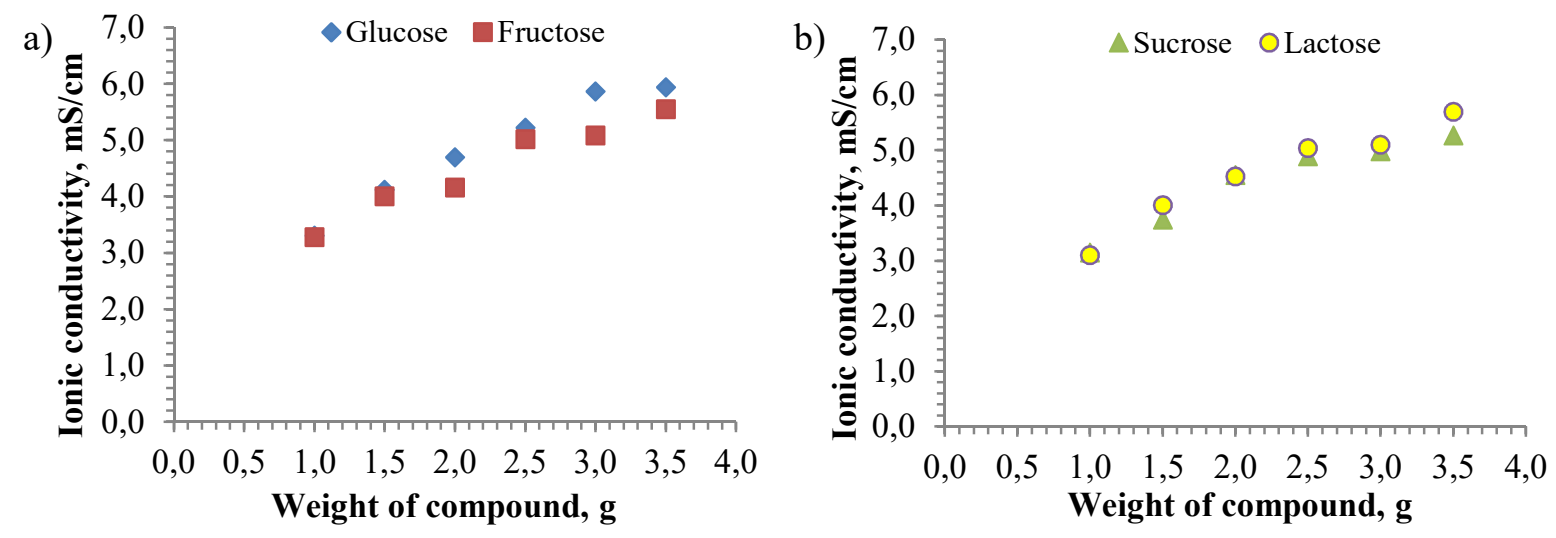

Figure 1. Ionic conductivity of (a) monosaccharide and (b) disaccharide with LiI in different weight of compound.

Figure 2 presents the effect of lithium iodide concentration on ionic conductivity. Figure showed ionic conductivity increased with addition of lithium iodide salt. The highest ionic conductivity for monosaccharide is $28.20 \mathrm{mS} / \mathrm{cm}$ for glucose with $35 \mathrm{wt} . \%$ of salt while for disaccharide is $28.00 \mathrm{mS} / \mathrm{cm}$ for lactose with $35 \mathrm{wt} . \%$ of salt at room temperature. According to Figure 2, it is confirmed that lithium iodide content has direct effect on ionic conductivity which dramatically increased the ionic conductivity in higher salt content. The reason is the number of $\mathrm{Li}^{+}$mobile ions in lithium iodide salts as charge carriers. Increase in salt concentration cause increase in number of $\mathrm{Li}^{+}$mobile ions.

a)

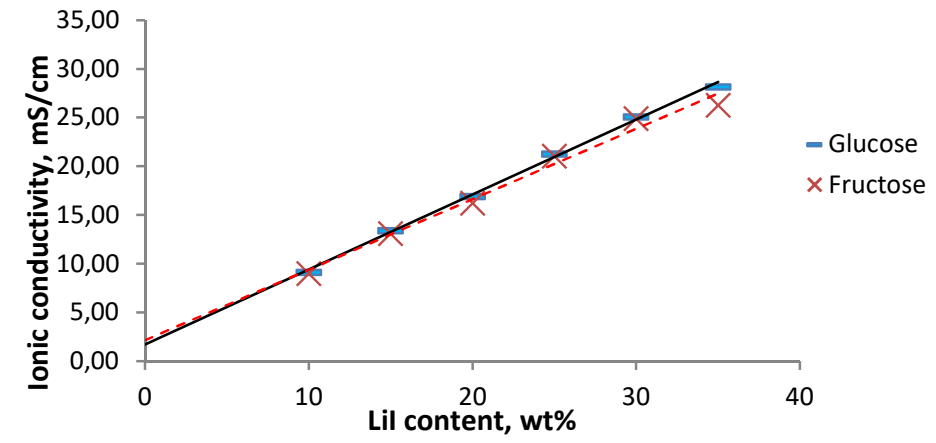

b)

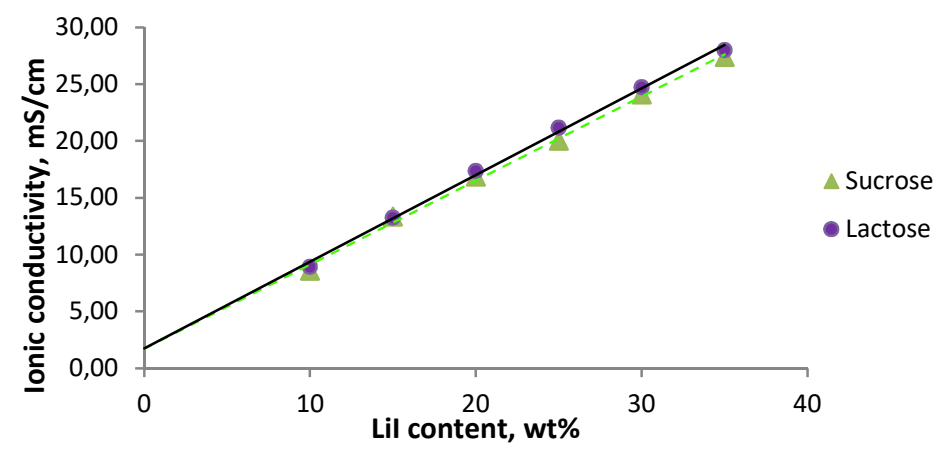

Figure 2. Ionic conductivity of (a) monosaccharide and (b) disaccharide with different LiI content. 


\subsection{Computational method}

\subsubsection{Optimization geometry}

Geometry optimization of glucose, fructose, sucrose and lactose were carried out in DFT using the B3LYP/6-31G ++ $(\mathrm{d}, \mathrm{p})$ basis set. The optimized structures of glucose, fructose, sucrose and lactose are shown in Figure 3. The optimisation geometry calculations locate the lowest energy molecular structure close to the specified starting structure.

Monosaccharides are glucose and fructose, they both have the same chemical formula, which is $\mathrm{C}_{6} \mathrm{H}_{12} \mathrm{O}_{6}$. Glucose ring contains 5 carbon atoms and one oxygen atom, while the fructose ring contains four carbon atoms and one oxygen atom. Before the optimization process, all the structures were planar with inaccurate bond lengths. As we can see in Figure 3, after the optimization process, the glucose structure shows a boat conformation while fructose structure shows an envelope conformation. The linear molecules' closure to make a ring creates a new chiral center at first carbon called anomeric carbon. It is caused by the position of hydrogen atom, hydroxyl group and methyl group $\mathrm{CH}_{2} \mathrm{OH}$ is in an axial or equatorial position with respect to the ring.

For disaccharides which are sucrose and lactose, they both also have the same chemical formula, which is $\mathrm{C}_{12} \mathrm{H}_{22} \mathrm{O}_{11}$. Sucrose is composed of glucose and fructose while lactose is composed of glucose and galactose. As we can see in Figure 3, for sucrose, one ring which is a glucose ring shown a chair conformation while the fructose ring shown like a planar. But when we rotated to other angles, fructose ring shown an envelope conformation. The position of hydrogen atom, hydroxyl group and methyl group $\mathrm{CH}_{2} \mathrm{OH}$ is staggered. For lactose, both monomer shown a boat conformation when we rotated to a variety of angles. From the figure, it is shown that ring of lactose in a boat conformation and the position of hydrogen atom, hydroxyl group and methyl group $\mathrm{CH}_{2} \mathrm{OH}$ is in an axial or equatorial position with respect to the ring.

(a)

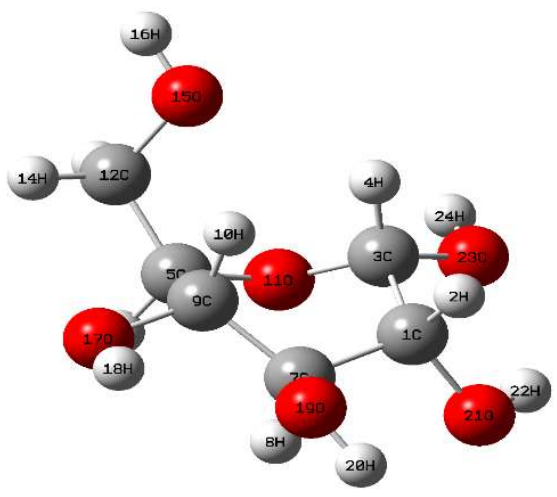

(c)

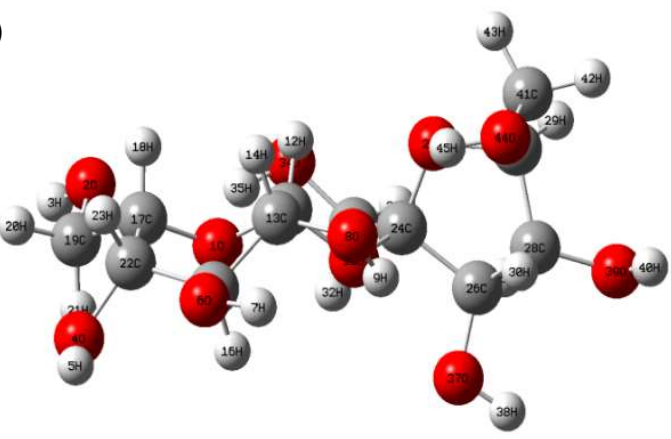

(b)
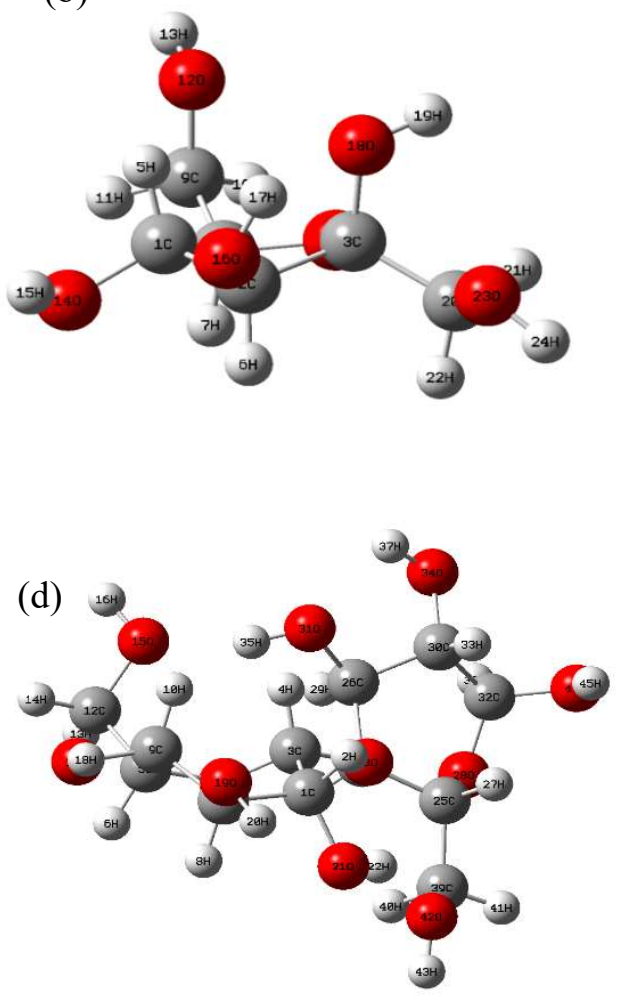

Figure 3. Optimized structure of (a) glucose (b) fructose (c) sucrose and (d) lactose calculated by DFT method using $6-31 \mathrm{G}++(\mathrm{d}, \mathrm{p})$ basis set. 


\subsubsection{Molecular electrostatic potential of glucose, fructose, sucrose and lactose}

Molecular electrostatic potential (MEP) is related to electron density. It is a very useful descriptor in understanding sites for hydrogen bonding interaction, nucleophilic and electrophilic reactions (Luque et al., $2000 \&$ Scrocco et al., 1979). To predict the reactive sites for electrophilic or nucleophilic attacks for the investigated molecule glucose, fructose, sucrose and lactose, MEP were calculated by using the B3LYP/6-31G ++ $(\mathrm{d}, \mathrm{p})$ basis set. Different colors show the different values of the electrostatic potential. The various colours on the surface are caused by different electrostatic potential values. The electrostatic potential rises in the following order: red $<$ orange $<$ yellow $<$ green $<$ blue indicating that it rises from the regions of most negative, most positive and zero electrostatic potential (Diego et al., 2015; Joshi \& Chaudhary, 2013).

The negative electrostatic potential corresponds to the proton's attraction by the aggregate electron density in the molecule (shade of red). In contrast, the positive electrostatic potential corresponds to a proton's repulsion by the atomic nuclei (shade of blue). The negative (red, orange and yellow) regions of MEP were related to electrophilic reactivity and the positive regions (green and blue) to nucleophilic reactivity. Figure 4 shows the MEP plot for glucose, fructose, sucrose and lactose calculated at B3LYP method.

From the MEP, it is evident that the negative charge (shade of red) covers much more the hydroxyl group and the positive charge (shade of blue) covers the region of hydrogen atom. According to previous studies, ionic conductivity can be measured when there is an interaction between ions with oxygen atom from functional group like hydroxyl. This is because oxygen atom have high electronegativity to interact with ions from salt (Imperiyka et al. 2014; Ahmad et al. 2011). The MEP confirmed that lithium ions can be interact with functional group of glucose, fructose, sucrose, and lactose. From the MEP, it is confirmed that lithium ions can be interact with hydroxyl group of glucose, fructose, sucrose and lactose which in shade of red. Thus, it will produce ionic conductivity.

(a)

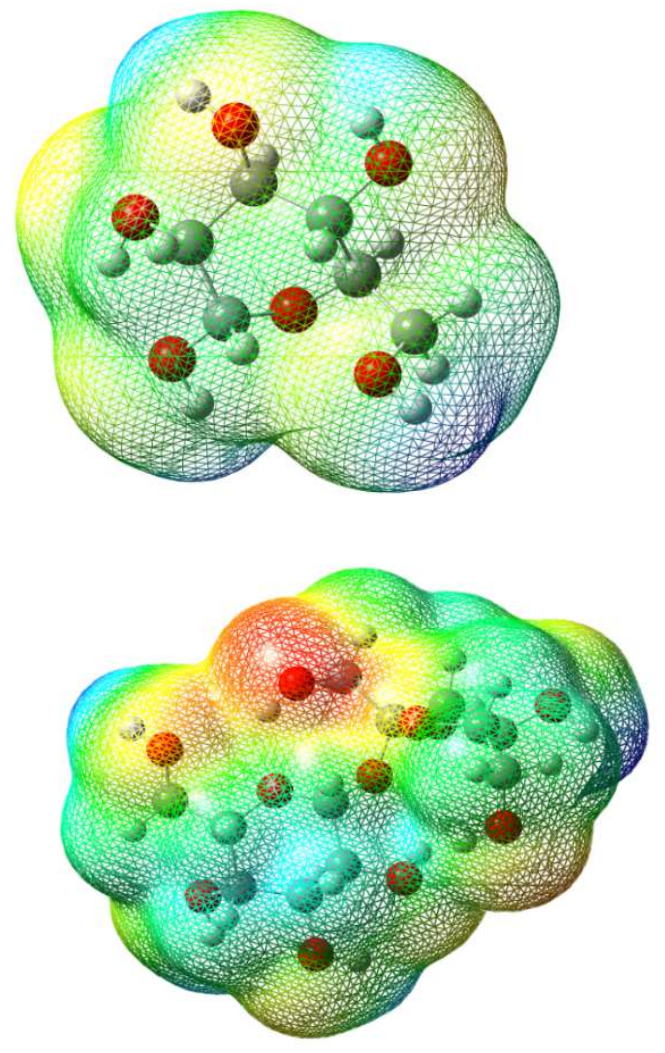

(b)
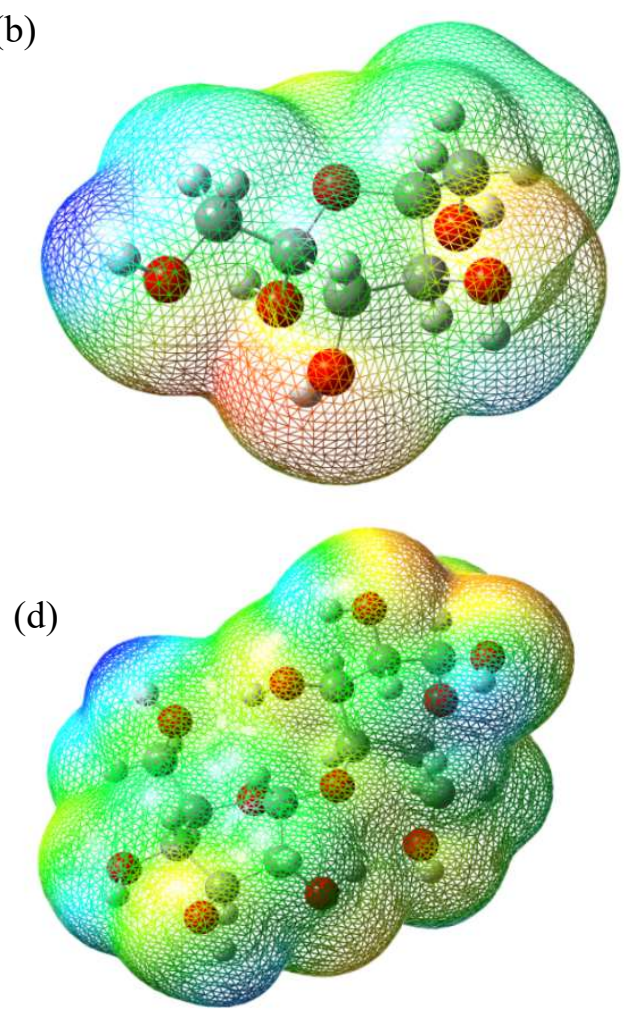

Figure 4. Molecular electrostatic potential (MEP) of (a) glucose (b) fructose (c) sucrose and (d) lactose calculated by DFT method using 6-31G ++ $(\mathrm{d}, \mathrm{p})$ basis set. 


\subsubsection{Mulliken charge analysis of glucose, fructose, sucrose and lactose}

Mulliken atomic charge calculation has an important role in the application of quantum chemical calculation to a molecular system due to the electric structure, dipole moment, atomic charge effect, molecular polarizability, and many more properties of molecular systems. These charges are expected to affect the properties, such as electronic parameters, refractivity, dipole moment and polarizability (Govindarajan et al. 2012). Mulliken atomic charges of glucose, fructose, sucrose and lactose calculated by using B3LYP/6-31G ++ (d, p) method are shown in Figure 5 and listed in Table 1.

As shown in Table 1, the positive charges are localized on the carbon and hydrogen atoms, while negative charges are localized on the certain carbon atom and all oxygen atoms. The analysis showed that the presence of the oxygen atom creates a negative charge on carbon atom. In glucose, the atom $\mathrm{O} 15$ shows the largest electronegativity with value $-0.562 \mathrm{e}$. In fructose, the atom $\mathrm{O} 12$ shows the largest electronegativity with value $-0.559 \mathrm{e}$. For disaccharide, in sucrose, the atom $\mathrm{O} 2$ shows the largest electronegativity with value $-0.515 \mathrm{e}$ while in lactose, the atom $\mathrm{O} 15$ shows the largest electronegativity with value $-0.567 \mathrm{e}$.

As we can see from the Table 1, for monosaccharide, oxygen atom in glucose shows the larger electronegativity compared to fructose. For disaccharide, lactose shows the larger electronegativity compared to sucrose. Different electronegativity exhibit different physical properties, including abilities to interact with other atoms such as lithium. Based on the ionic conductivity result, glucose and lactose produced higher ionic conductivity compared to fructose and sucrose. From the Mulliken charge analysis, glucose and lactose also showed largest electronegativity than fructose and sucrose. It is reveals that electronegativity value of each monomer effect the ionic conductivity of electrolyte. Thus, it can be concluded that the larger the electronegativity value of oxygen on the monomer, the greater tendency an oxygen atom has to attract to a lithium ions, the higher ionic conductivity of electrolyte system.

(a)

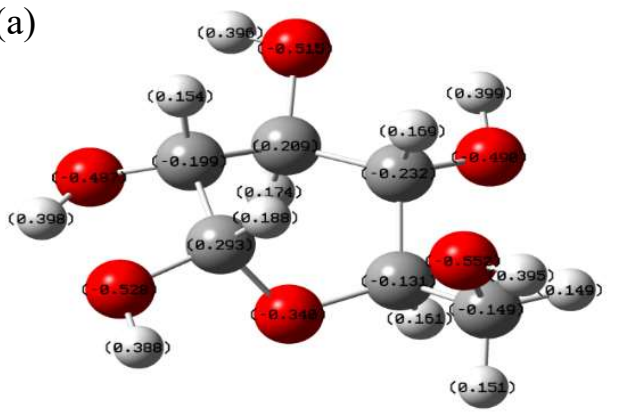

(c)

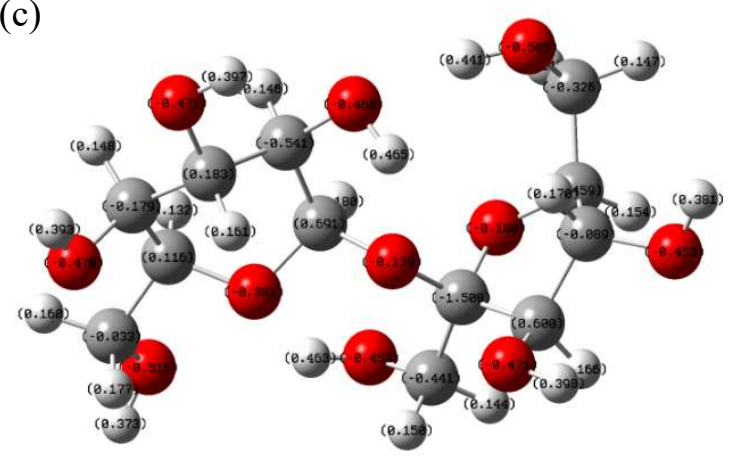

(b)

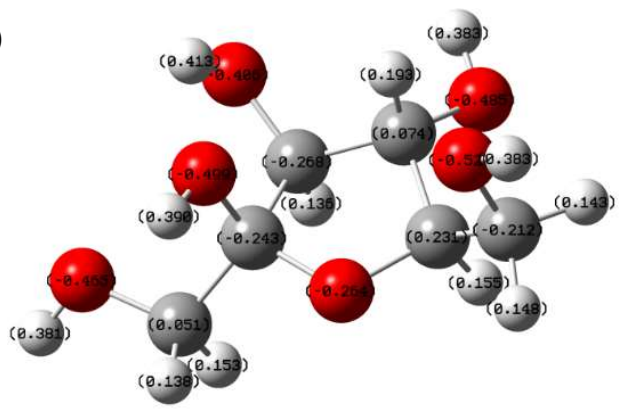

(d)

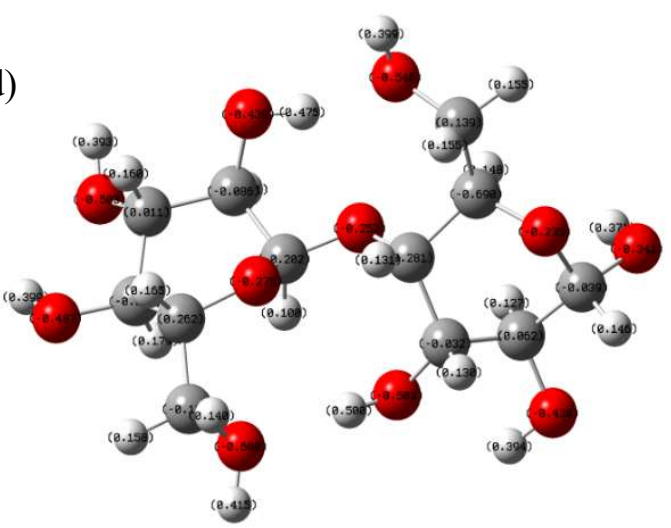

Figure 5. Mulliken atomic charge distribution of (a) glucose (b) fructose (c) sucrose and (d) lactose calculated by DFT method using 6-31G $++(d, p)$ basis set. 
Ra'il and Mobarak: Effect of Monosaccharides and Disaccharides Type on Ionic Conductivity

Table 1 Mulliken charges of glucose, fructose, sucrose and lactose calculated by DFT using 6-31G ++ (d,p) basis set

\begin{tabular}{cccc}
\hline \multicolumn{4}{c}{ DFT/B3LYP 6-31G ++ (d, p) } \\
\hline Atom & Glucose & Atom & Fructose \\
\hline O 11 & -0.339887 & O 8 & -0.264120 \\
O 15 & -0.551552 & O 12 & -0.528929 \\
O 17 & -0.489584 & O 14 & -0.484776 \\
O 19 & -0.515242 & O 16 & -0.405800 \\
O 21 & -0.487382 & O 18 & -0.498807 \\
O 23 & -0.528300 & O 23 & -0.464648 \\
\hline & Disaccharide & \\
\hline Atom & Sucrose & Atom & Lactose \\
\hline O 1 & -0.381102 & O 11 & -0.274928 \\
O 2 & -0.515079 & O 15 & -0.608030 \\
O 4 & -0.470361 & O 17 & -0.487351 \\
O 6 & -0.471050 & O 19 & -0.503033 \\
O 8 & -0.459686 & O 21 & -0.438606 \\
O 10 & -0.138901 & O 23 & -0.252992 \\
O 25 & -0.107749 & O 28 & -0.234536 \\
O 34 & -0.452349 & O 31 & -0.503451 \\
O 37 & -0.471150 & O 34 & -0.438180 \\
O 39 & -0.452711 & O 42 & -0.546079 \\
O 44 & -0.505129 & O 44 & -0.341496 \\
\hline
\end{tabular}

\subsubsection{Interaction between glucose and lactose with lithium}

Glucose has 6 oxygen atoms while lactose has 11 oxygen atoms which are conveniently positioned to interact with the metal. Figure 6 shows the optimization structure of glucose and lactose interact with lithium that carried out in DFT using the B3LYP/6-31G $++(d, p)$ basis set, while Table 2 shows the bond length and binding energy of glucose and lactose with lithium. Each atom oxygen in glucose and lactose have different electronegativity. It is cause the difference tendency of oxygen atom has to attract to a lithium. No previous research stated which functional groups in a monomer that favours to interact with ions from salt. This research presents the discovery on functional groups that tend to interact with ions and cause the ionic conductivity in electrolyte system.

Electronegativity of oxygen atom in glucose decrease in the order O-15 $>0-23>0-19>0-17$ $>\mathrm{O}-21>\mathrm{O}-11$ while in lactose decrease in the order O-15 $>\mathrm{O}-42>\mathrm{O}-31>\mathrm{O}-17>\mathrm{O}-11>\mathrm{O}-28$. But as shown in Table 2, the bond length of oxygen atom with lithium for glucose increase in the order O$15<\mathrm{O}-23<\mathrm{O}-21<\mathrm{O}-19<\mathrm{O}-11<\mathrm{O}-17$ while in lactose increase in the order O-17<0-42<0-31< $\mathrm{O}-28<\mathrm{O}-11<\mathrm{O}-15$. In glucose, $\mathrm{O}-15$ has the highest electronegativity which means it has greater tendency to attract to a lithium ions. From the bond length and binding energy in Table 2, even it has low binding energy, it has the shorter bond length. It is confirmed that $\mathrm{O}-15$ has greater tendency to attract with lithium ions due to the shorter bond length. As we can see, $\mathrm{O}-21$ has less electronegativity than $\mathrm{O}-19$ but O-21 has shorter bond length than O-19. This is because from Table 2, O-21 shows it has higher binding energy compared to O-19. For O-17, it has greater electronegativity and higher binding energy than O-11, but O-11 has the shorter bond length than O-17. It is because O-17 and O-19 interact with Li-25 together as shown in Figure 6(c). The O-19 has greater electronegativity compared to O-17, it is caused O-17 has longer bond length as O-19 has higher tendency to attract to a lithium.

In lactose, $\mathrm{O}-15$ has the highest electronegativity to attract to a lithium ions. But from the bond length as shown in Table 2, O-15 has the longest bond length with lithium because O-15 and O-31 interact with Li-46 together as shown in Figure 6(f). When both oxygen interact with same lithium, it is caused $\mathrm{O}-15$ has longest bond length. For O-42, it has greater electronegativity than $\mathrm{O}-17$ but $\mathrm{O}-17$ has 
shorter bond length with lithium compared to O-42. It is because O-42 and O-11 interact with Li-46 together as shown in Figure 6(e). When two oxygen atoms interact with same lithium ion, it will effect the bond length. For O-17, it is only one oxygen atom interact with lithium ion, thus O-17 has shorter bond length than O-42. For O-11, it has shorter bond length than O-15 even O-15 has greater electronegativity compared to $\mathrm{O}-11$. It is because $\mathrm{O}-11$ has higher binding energy to interact with lithium compared to O-15.

From the optimize structural parameters such as bond lengths, molecular electrostatic potential (MEP), mulliken charge analysis and binding energy of interaction with lithium, it can be concluded that functional groups dominant when interact with lithium not only depends on electronegativity value but also strength of binding energy to attract ions.

(a)

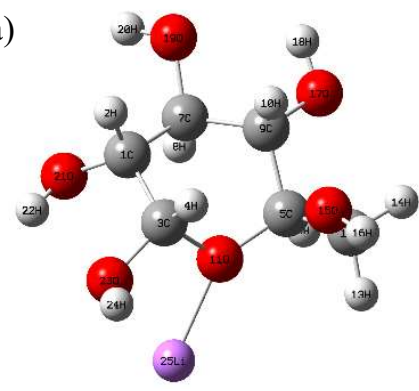

(d)

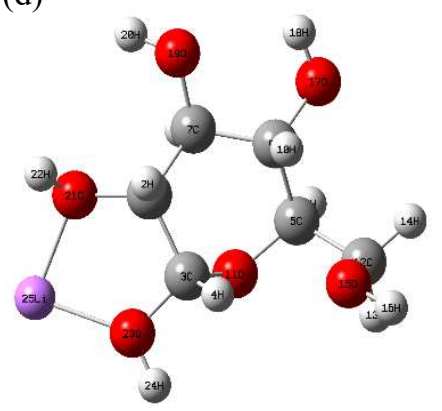

(b)

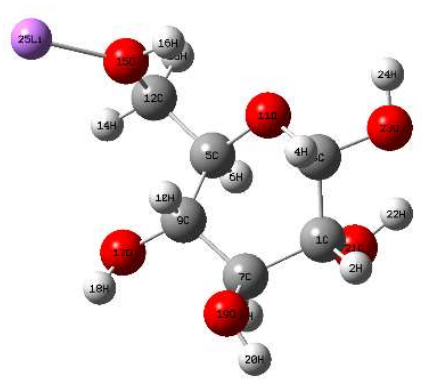

(e)

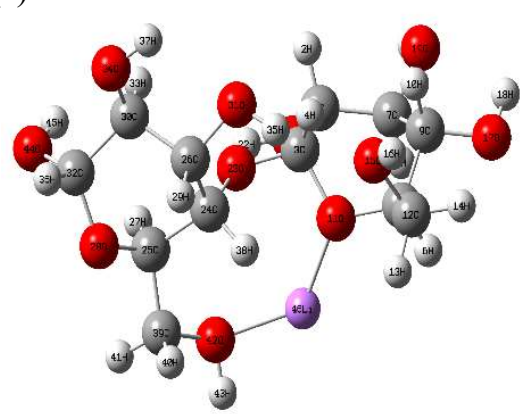

(c)

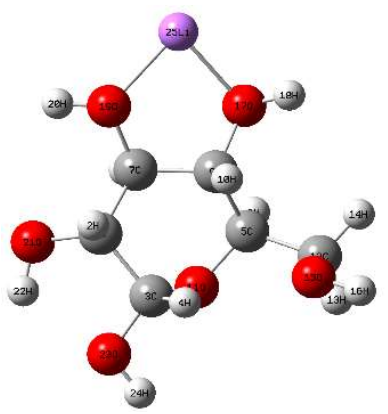

(f)

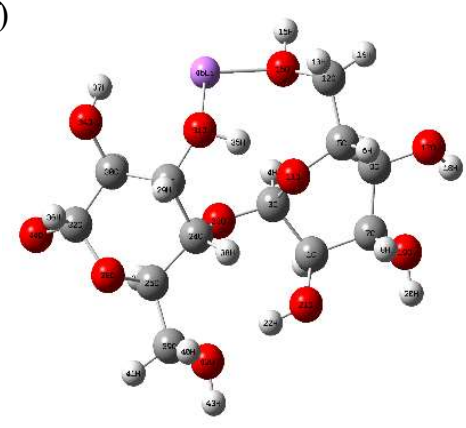

(g)

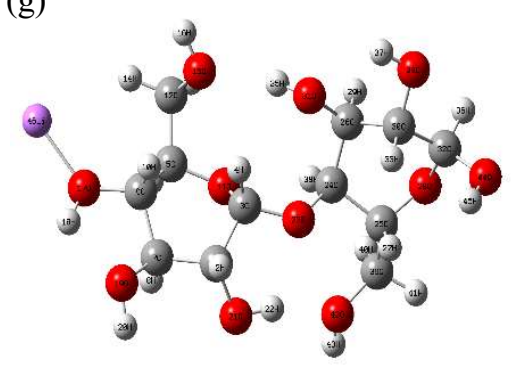

(h)

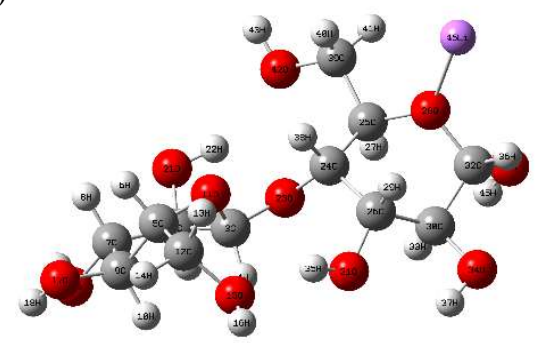

Figure 6. Optimized structure interaction of (a) glucose O11 - Li25; (b) glucose O15 - Li25; (c) glucose O17 Li25 - O19; (d) glucose O21 - Li25 - O23; (e) lactose O11 - Li46 - O42; (f) lactose O15 - Li46 - O31; (g) lactose O17 - Li46; and (h) lactose O28 - Li46 calculated by DFT method using 6-31G ++ (d, p) basis set. 
Ra'il and Mobarak: Effect of Monosaccharides and Disaccharides Type on Ionic Conductivity

Table 2 Bond length and binding energy of glucose and lactose calculated by DFT using 6-31G $++(\mathrm{d}$, p) basis set

\begin{tabular}{|c|c|c|c|c|c|}
\hline \multicolumn{6}{|c|}{ DFT/B3LYP 6-31G ++ (d, p) } \\
\hline \multicolumn{3}{|c|}{ Glucose } & \multicolumn{3}{|c|}{ Lactose } \\
\hline Atom & $\begin{array}{c}\text { Bond length } \\
(\AA)\end{array}$ & $\begin{array}{c}\text { Binding energy } \\
(\mathrm{eV})\end{array}$ & Atom & $\begin{array}{c}\text { Bond length } \\
(\AA)\end{array}$ & $\begin{array}{c}\text { Binding energy } \\
(\mathrm{eV})\end{array}$ \\
\hline O $11-\mathrm{Li} 25$ & 1.96023 & -6.360391462 & O $11-\mathrm{Li} 46$ & 1.96172 & -6.615527443 \\
\hline O $15-\mathrm{Li} 25$ & 1.88576 & -6.360473368 & O $15-\mathrm{Li} 46$ & 1.97573 & -6.574957969 \\
\hline O $17-\mathrm{Li} 25$ & 1.97045 & -6.495433199 & O $17-\mathrm{Li} 46$ & 1.91376 & -7.248543904 \\
\hline O $19-\mathrm{Li} 25$ & 1.94983 & -6.495433199 & O $28-\mathrm{Li} 46$ & 1.94493 & -7.163146098 \\
\hline O $21-\mathrm{Li} 25$ & 1.92849 & -6.531560685 & O $31-\mathrm{Li} 46$ & 1.93212 & -6.574957969 \\
\hline O $23-$ Li 25 & 1.91504 & -6.531560685 & O $42-\mathrm{Li} 46$ & 1.92922 & -6.615527443 \\
\hline
\end{tabular}

\section{CONCLUSION}

Liquid electrolytes based monosaccharide and disaccharide with lithium iodide were prepared and interaction between compound and lithium iodide occurred.The addition of lithium salts increases the density of mobile ions, thus enhancing ionic conductivity.The highest ionic conductivity for monosaccharide is glucose at $28.20 \mathrm{mS} / \mathrm{cm}$ while for disaccharide is lactose at $28.00 \mathrm{mS} / \mathrm{cm}$ with percentage of salt at $35 \mathrm{wt} . \%$. The computer simulations of DFT showed that interaction between lithium with compounds can be occurred due to negative electrostatic potential on the molecule. Electronegativity value of oxygen atom in glucose $(-0.562 \mathrm{e})$ and lactose $(-0.567 \mathrm{e})$ higher than fructose $(-0.559 \mathrm{e})$ and sucrose $(-0.515 \mathrm{e})$. Functional groups that are dominant to interact when interact with lithium salt are $\mathrm{O}-15$ for glucose and $\mathrm{O}-17$ for lactose due to the shorter bond length, the stronger energy attraction between functional groups with lithium ion.

\section{ACKNOWLEDGEMENT}

This work was subsidized with grants from GUP-2018-036. We gratefully thank you our colleges from Faculty Science and Technology who provided inside and expertise that greatly assisted the research. We also would like to thank Prof. Madya Dr. Ku Halim Ku Bulat from Universiti Malaysia Terengganu for providing the Gaussian software.

\section{REFERENCE}

Ahmad, A., Rahman, M. Y. A., Low, S. P., \& Hamzah, H. (2011). Effect of $\mathrm{LiBF}_{4}$ salt concentration on the properties of plasticized MG49-TiO2 based nanocomposite polymer electrolyte. International Scholarly Research Notices.

Aziz, S. B., \& Abidin, Z. H. Z. (2013). Electrical conduction mechanism in solid polymer electrolytes: new concepts to arrhenius equation. Journal of Soft Matter, 2013, 1-8.

Benhamou, K., Kaddami, H., Magnin, A., Dufresne, A., \& Ahmad, A. (2015). Bio-based polyurethane reinforced with cellulose nanofibers: a comprehensive investigation on the effect of interface. Carbohydrate Polymers, 122, 202-211.

Gil, D. M., Lestard, M. D., Estévez-Hernández, O., Duque, J., \& Reguera, E. (2015). Quantum chemical studies on molecular structure, spectroscopic (IR, Raman, UV-Vis), NBO and Homo-Lumo analysis of 1benzyl-3-(2-furoyl) thiourea. Spectrochimica Acta Part A: Molecular and Biomolecular Spectroscopy, 145, 553-562.

Govindarajan, M., Karabacak, M., Udayakumar, V. \& Periandy, S. (2012). FTIR, FTRaman and UV Spectral Investigation: Computed Frequency Estimation Analysis and Electronic Structure Calculations on Chlorobenzene Using HF and DFT. Spectrochimica Acta Part A: Molecular and Biomolecular Spectroscopy, 88, 37-48. 
Imperiyka, M., Ahmad, A., Hanifah, S. A., \& Rahman, M. Y. A. (2014). Preparation and characterization of polymer electrolyte of glycidyl methacrylate-methyl methacrylate- $\mathrm{LiClO}_{4}$ plasticized with EC. International Journal of Polymer Science, 2014-1, 371-377.

Joshi, B. D., \& Chaudhary, P. N. (2013). Molecular structure, MESP, homo-lumo and vibrational analysis of $\beta$ asarone using density functional theory. Kathmandu Univ J Sci Eng Tech, 9, 1-14.

Khanmirzaei, M. H., \& Ramesh, S. (2013). Ionic transport and FTIR properties of lithium iodide doped biodegradable rice starch-based polymer electrolytes. Int J Electrochem Sci, 8(7), 9977-9991.

Liu, K., Lin, Y., Miller, J. D., Liu, J. \& Wang, X. (2017). Study of Sucrose Based Room Temperature Solid Polymer Electrolyte for Lithium Sulfur Battery. Journal of The Electrochemical Society, 164 (2), 447452.

Luque, F. J., López, J. M. \& Orozco, M. (2000). Perspective on Electrostatic Interactions of a Solute with a Continuum. A Direct Utilization of Ab Initio Molecular Potentials for the Prevision of Solvent Effects. Theoretical Chemistry Accounts, 103 (3-4), 343-345.

Mobarak, N. N., Ramli, N., Ahmad, A. \& Rahman, M. Y. A. (2012). Chemical Interaction and Conductivity of Carboxymethyl K-Carrageenan Based Green Polymer Electrolyte. Solid State Ionics, 224, 51-57.

Scrocco, E. \& Tomasi, J. (1978). Electronic Molecular Structure, Reactivity and Intermolecular Forces: An Euristic Interpretation by Means of Electrostatic Molecular Potentials. Advances in Quantum Chemistry, 11, 115-193.

Selvin, P. C., Perumal, P., Selvasekarapandian, S., Monisha, S., Boopathi, G., \& Chandra, M. L. (2018). Study of proton-conducting polymer electrolyte based on K-carrageenan and $\mathrm{NH}_{4} \mathrm{SCN}$ for electrochemical devices. Ionics, 24(11), 3535-3542.

Zulkefli, F. N., Navaratnam, S., \& Ahmad, A. H. (2015). Proton conducting biopolymer electrolytes based on starch incorporated with ammonium thiocyanate. Advanced Materials Research, 1112, 275-278.

Wade, L. G. (2014). Organic chemistry. International Editions, 1174-1178, Pearson, London. 